\title{
SAR backscatter multitemporal compositing via local resolution weighting
}

\author{
Small, David
}

\begin{abstract}
A method is presented for generating composite SAR imagery from a set of multiple radiometrically terrain-corrected (RTC) backscatter images and their co-registered local illuminated areas. The process is implemented in map geometry: the resolution of each image is estimated locally as the inverse of the local contributing area and used to weight the contributions from all available observations. Some potential contributors could be out-of-swath or occluded by radar shadow. The resulting composite backscatter image can trade off temporal resolution for improved local spatial resolution while simultaneously increasing the local number of looks. The composite image is characterized by more homogenous properties (resolution, noise) in comparison to single acquisitions. The technique is demonstrated using ScanSAR data from ENVISAT ASAR and Radarsat-2.
\end{abstract}

DOI: https://doi.org/10.1109/IGARSS.2012.6350465

Posted at the Zurich Open Repository and Archive, University of Zurich ZORA URL: https://doi.org/10.5167/uzh-68085

Conference or Workshop Item

Published Version

Originally published at:

Small, David (2012). SAR backscatter multitemporal compositing via local resolution weighting. In: Geoscience and Remote Sensing Symposium (IGARSS), 2012 IEEE International, Munich, Germany, 22 July 2012 - 27 July 2012. IEEE International, 4521-4524.

DOI: https://doi.org/10.1109/IGARSS.2012.6350465 


\title{
SAR BACKSCATTER MULTITEMPORAL COMPOSITING VIA LOCAL RESOLUTION WEIGHTING
}

\author{
David Small \\ Remote Sensing Laboratories, Dept. of Geography, University of Zurich \\ Winterthurerstrasse 190, CH-8057 Zurich, Switzerland; E-mail: david.small@ geo.uzh.ch
}

\begin{abstract}
A method is presented for generating composite SAR imagery from a set of multiple radiometrically terraincorrected (RTC) backscatter images and their co-registered local illuminated areas. The process is implemented in map geometry: the resolution of each image is estimated locally as the inverse of the local contributing area and used to weight the contributions from all available observations. Some potential contributors could be out-of-swath or occluded by radar shadow. The resulting composite backscatter image can trade off temporal resolution for improved local spatial resolution while simultaneously increasing the local number of looks. The composite image is characterized by more homogenous properties (resolution, noise) in comparison to single acquisitions. The technique is demonstrated using ScanSAR data from ENVISAT ASAR and Radarsat-2.
\end{abstract}

Index Terms - SAR, Terrain, Geometry, Radiometry, Radar terrain factors

\section{INTRODUCTION}

Increasingly robust experience with modern radar sensors leads to reconsideration of previously established understanding of the spatial and temporal resolutions of SAR images. The theoretical definition of SAR spatial resolution was adjusted in [19]. Canonical backscatter normalisation conventions were revised in [12]. This paper considers a method to trade off spatial and temporal resolutions to create low-noise composite imagery with relatively homogenous image properties in comparison to a single data take. It weighs each contribution to the composite by the local resolution in each input image, offering optimal use of ascending and descending imagery.

Both the geometry and radiometry of SAR imagery is severely distorted in regions with terrain undulations present. Conventional terrain-geocoding solves the Doppler and range equations to transform image content from radar geometry (a slant or ground-range product) into a map geometry (e.g. UTM). When the SAR sensor provides highly accurate state vectors, radar timing annotations, and geometric resolution, it becomes worthwhile to model and correct for additional path delay corrections due to atmospheric effects, solid Earth tides [9], and integrated plate tectonics [10].

Geometric Terrain Correction, that is the generation of higher level "GTC" products, transforms SAR data from the sensor's native radar geometry into map coordinates however, unless further steps are taken, the effects of terrain on the spatial distribution of the image radiometry, i.e. the backscatter estimates themselves remain present. The backscatter can be represented as beta [7], sigma, or gamma nought: the equations underlying the geometric transformations behind GTC production are unaffected by the choice. After resampling in map geometry, the GTC backscatter estimates inherently contain a mixture of terrain effects and the land cover signatures under study. To improve the quality of the land cover signature, radiometric terrain correction is applied by modelling the effects of terrain on the backscatter and then applying compensation to "level the playing field", flattening the backscatter amplitude. After the flattening step, a single land cover class should manifest relatively consistent behaviour independent of the local terrain-slope. Widespread methodologies typically map terrain effects on backscatter homomorphically between the radar \& DHM geometries, using slope angles (in the range and azimuth dimensions as described e.g. in [18]) and then apply compensation. A more robust technique has been developed recently that ignores slope angles and instead adds together all contributions of the finite DHM facet areas that are applicable at each radar geometry azimuth and range coordinate [14].

\section{TERRAIN-FLATTENED GAMMA NOUGHT}

When the definition of the areas used for backscatter normalisation adheres strictly to the $\gamma^{0}$ convention, i.e. the area is expressed in the plane perpendicular to the local slant range direction, and when occlusions (radar shadow) are systematically excluded from contributing, then the actual area $A_{\gamma}$ visible to the radar at each azimuth $j$ and range coordinate $i$ can be estimated using a DHM: the resulting 
image simulation is then referred to as $A_{\gamma}(i, j)$. Flattening is then easily applied by dividing the backscatter measurement beta nought $\beta^{0}(i, j)$ by the local contributing area $A_{\gamma}(i, j)$ a detailed description of the methodology is provided in [14]. The resulting backscatter value is known as the flattened radar backscatter parameter "terrain-flattened gamma nought" [12]:

$$
\gamma_{T}^{0}(i, j)=\beta^{0}(i, j) \cdot A_{\beta} / A_{\gamma}(i, j)
$$

An example of an ascending/descending (A/D) overlay is presented in [15]. Although the method generally performs

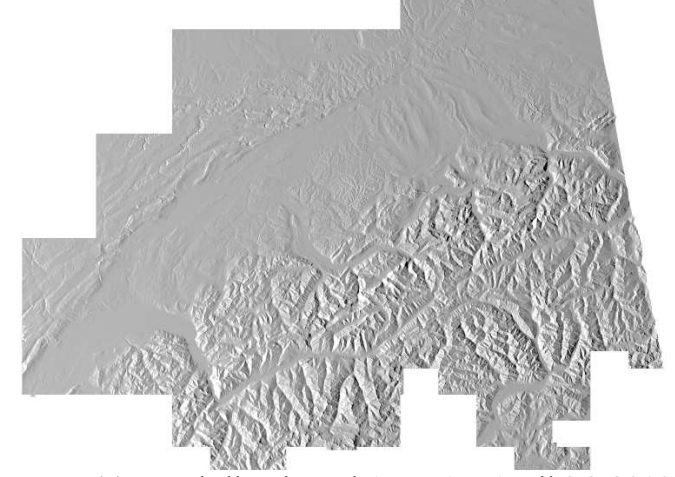

(a) Local Illuminated Area $A \gamma$-April 26, 2010 17:23

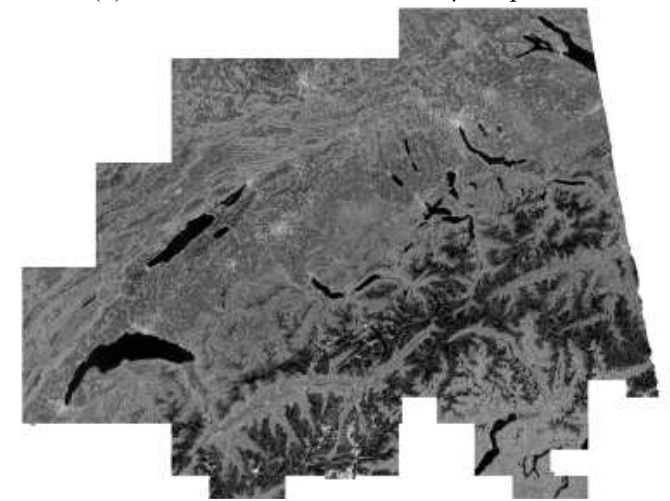

(c) RTC $\gamma_{T}^{0}-$ April 26, 2010 17:23

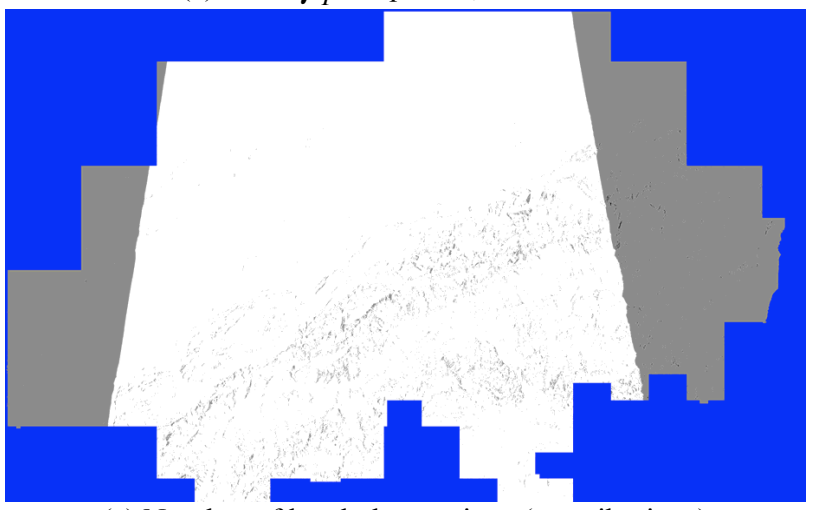

(e) Number of local observations (contributions) well in flattening the radiometry of images acquired even in steep terrain such as the Swiss Alps, it is unable to improve upon the locally available geometric resolution at each coordinate. The locally available resolution can be understood as the reciprocal of the local contributing area: widespread contributions (e.g. on foreslopes) imply poor local resolution, while an "exclusively minute" contributing area (e.g. on backslopes) implies extremely high local resolution. All terrain corrections shown here were performed using swisstopo's DHM25 height model [16].

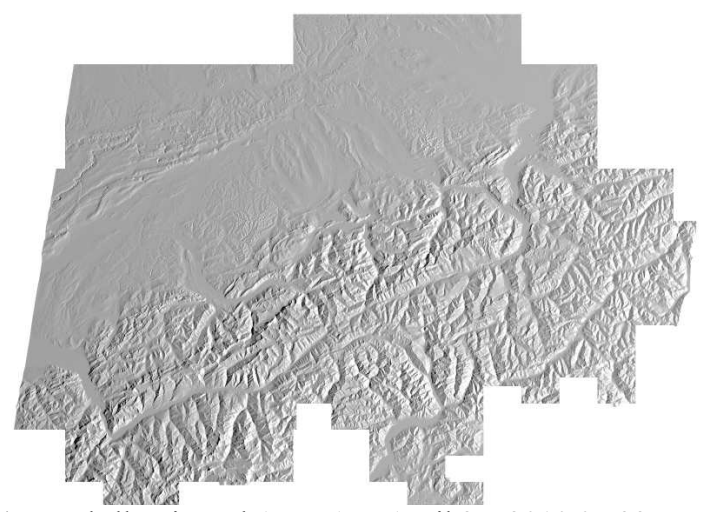

(b) Local Illuminated Area $A \gamma$ - April 27, 2010 05:33

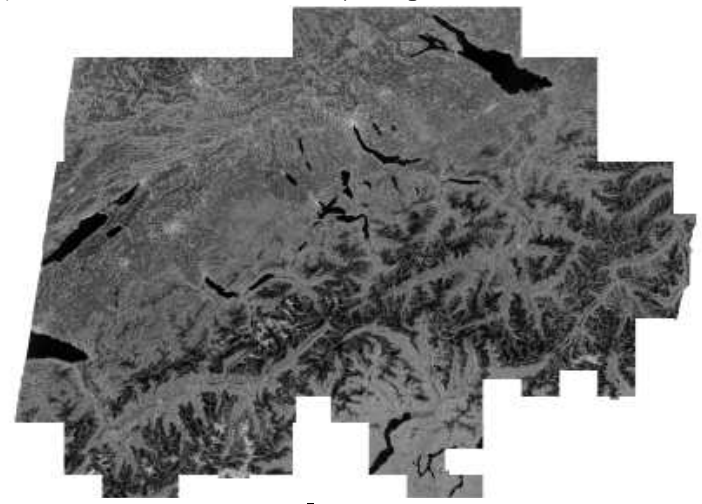

(d) Descending RTC r $;$ - April 27, 2010 05:33

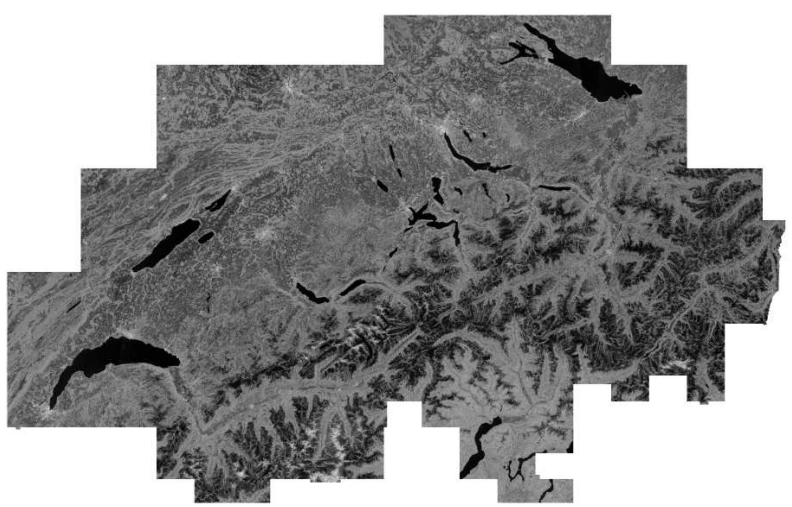

(f) Local Resolution Weighted Backscatter $r$ :':" :

Figure 1: Local Resolution Weighting applied to Radarsat-2 ScanSAR narrow VH-pol imagery of Switzerland - (a) Local Illuminated Area $A \gamma$ April 26; (b) Local Illuminated Area $A \gamma$ April 27; (c) RTC $\gamma_{T}^{0}$ April 26; (d) RTC $\gamma_{T}^{0}$ April 27; (e) Number of contributions (blue: outside DHM/none, black: shadowed/none, grey: 1, white: 2); (f) Local resolution-weighted Backscatter $\gamma_{L R W}^{0}{ }^{--}$ black: -26dB, white: $-1 \mathrm{~dB}$; swisstopo DHM25 (25m postings) was used for terrain corrections 


\section{LOCAL RESOLUTION WEIGHTING}

In backscatter amplitude-based land cover classification, the class separability is determined by the noise present in combination with the radiometric stability and absolute radiometric calibration [4]. Terrain-induced backscatter variability directly impacts classification accuracy [1], as does the noise level present in the backscatter estimates. Multi-temporal SAR backscatter interpretation has traditionally either avoided regions with hilly terrain, or restricted itself to repeat-pass single-track time series, selecting "practically identical geometries" [6]. Single-track processing then typically proceeds with slope effects neglected: "...without correcting for the effect of the local terrain slope on the pixel area ... as ... correction factors remain the same across all images" [2]. In this work, singletrack restrictions are lifted: application of slope corrections opens a world of multi-track backscatter synergies.

As originally proposed in [11], one can partially compensate for the effects of spatially varying local resolution by combining ascending and descending views of the same scene, trading off improvements in spatial resolution for a slight blurring in temporal focus. Regions viewed as foreslopes in an asc. image will tend to be seen as backslopes in a desc. product, so combinations of asc./desc. products produce particular hybrid vigour.

Given that the local illuminated area is inversely proportional to the local image resolution, multiple RTC $\gamma_{T}^{0}$ backscatter estimates may be combined to advantage by weighing each individual contribution by its known local resolution. Given a set of $N$ RTC images, at each individual point, the number of available contributions $M$ will be less than or equal to $N$. It can be less due to either lack of local coverage (outside swath) or radar shadow. The weight assigned to an image $i$ within the set of $M$ is then:

$$
W_{i}=\frac{1}{A_{i}} / \sum_{j=1}^{M} \frac{1}{A_{j}} .
$$

The LRW composite backscatter is then computed as the weighted sum of the flattened backscatter observations:

$$
\gamma_{L R W}^{0}=\sum_{i=1}^{M} W_{i} \cdot \gamma_{i}^{0}
$$

Images with the highest available local resolution receive the highest local weights. For example, the composite of a region imaged as a backslope in an asc. image, likely seen as a foreslope in a desc. acquisition, will receive a far greater contribution from the asc. than the desc. backscatter measurement. Note however that all available observations do contribute, reducing noise and increasing the equiv. number of looks (ENL).

First results produced using Envisat ASAR wide swath (WS) imagery were reported in [12]. In this paper, local resolution weighting is applied to further ASAR data, as well as dual-pol observations made with Radarsat-2 (R2). Two R2 ScanSAR narrow (SCN) images of Switzerland were acquired within 12 hours of each other on April 26-27, 2010. Terrain-flattening [14] was applied to the VV \& VH backscatter measurements and reported in [15]. The products are used in this paper to illustrate application of the local-resolution-weighting (LRW) technique.

The LRW process is demonstrated in Figure 1. The local illuminated areas computed during the radiometric terrain correction are shown in (a) and (b). The original ground range versions and geometrically-terrain-corrected (GTC) backscatter values can be seen in [15]. The RTC $\gamma_{T}^{0}$ estimates for the asc. \& desc. acquisitions are shown in (c) and (d) respectively. The number of observations available locally is mapped in (e): blue specifies that the region is outside of the DHM used for terrain-correction. Grey indicates that only a single observation is available locally due to shadow or swath limitations. In this case, the weight of the single observation reduces to unity. White indicates that two observations are available locally. The LRW composite backscatter image itself is shown in Figure 1(f). Residual effects of foreshortening, layover, or shadow are difficult to find, even in regions of particularly steep terrain (e.g. the canton of Valais in the SW). More spatially homogenous image properties simplify image interpretation.

Two ASAR wide-swath (WS) LRW composites are shown in Figure 2. In a manner similar to Figure 1(e), the number of local contributions is mapped in a small inset at the lower right. Both composite backscatter maps were generated via LRW using asc./desc. observations acquired within a single day by ASAR. Although each single RTC image provided only partial coverage of Switzerland, the LRW composite delivers full coverage with added value (higher resolution \& lower noise) in the overlap region.

The ASAR WS products used as input for Figure 2(b) were acquired by ENVISAT on April 7, 2012, the day before contact was lost with the satellite. The image illustrates low backscatter from wet snow at intermediate heights in the Alps. Note that no "interstrip mosaic balancing" compensation (e.g. as in [5]) is applied at the swath edges. Although no "feathering" of any type is employed, the beam boundary is hard to spot unless water bodies with differing amounts of Bragg scattering are crossed or resolution / ENL variations across the composite become prominent.

\section{CONCLUSIONS}

The combination of local resolution weighting and terrainflattened $\gamma_{T}^{0}$ backscatter values promises to enable the generation of higher level SAR products with more spatially consistent properties: both local geometric resolution and the number of local looks improve. The composite is more homogenous spatially than is the case in any single contributing product. LRW products could be used for land cover monitoring, e.g. the mapping of wet snow cover in Alpine environments - e.g. with $\mathrm{CoReH} 2 \mathrm{O}$ [8]. The ESA Sentinel-1 satellites [17] and the Radarsat Constellation 
Mission [3] (in isolation or in combination) should offer sufficiently frequent revisit times that some temporal resolution could conceivably be traded for improved image properties in higher level composite backscatter products. Optimising the trade-off between improved spatial resolution and image homogeneity vs. slightly blurred temporal focus will be the subject of future research.

\section{ACKNOWLEDGMENTS}

The Radarsat-2 data was provided by the Canadian Space Agency through its SOAR programme (project 1985). RADARSAT-2 Data and Products (C) MacDONALD, DETTWILER AND ASSOCIATES LTD. (2010) - All Rights Reserved. The ASAR data was provided by ESA. The digital height model DHM25 from swisstopo was used for all terrain corrections.

\section{REFERENCES}

[1] Atwood D., D. Small, and R. Gens, "Improving PolSAR Land Cover Classification with Radiometric Correction of the Coherency Matrix," IEEE J. of Selected Topics in Applied Remote Sensing, 2012 (in press).

[2] Bruzzone L., M. Marconcini, U. Wegmüller, and A. Wiesmann, "An advanced system for the automatic classification of multitemporal SAR images," IEEE Trans. Geosci. Remote Sens, 42(6), pp. 1321-1334, Jun. 2004.

[3] Colinas J., G. Seguin, and P. Plourde, "Radarsat Constellation, moving toward implementation," Proc. IGARSS 2010, Honolulu, USA, pp. 3232-3235.

[4] Davidson M., E. Attema, N. Floury, B. Rommen, P. Snoeij, "A Closed-form Expression Relating Classification Accuracy to SAR System Calibration Uncertainty," IEEE Geosci. \& Remote Sensing Letters, 6(3), pp. 467-470, July 2009.

[5] De Grandi G., A. Bouvet, R. M. Lucas, M. Shimada, S. Monaco, and A. Rosenqvist, "The K\&C PALSAR Mosaic of the African Continent: Processing Issues and First Thematic Results," IEEE Trans. Geosci. Remote Sens, 49(10), pp. 3593-3610, Oct. 2011.

[6] Quegan S., T. Le Toan, J. J. Yu, F. Ribbes, and N. Floury, "Multitemporal ERS SAR analysis applied to forest mapping," IEEE Trans. Geosci. Remote Sens, 38(2), pp. 741753, Mar. 2000.

[7] Raney K., A. Freeman, B. Hawkins, and R. Bamler, "A Plea for Radar Brightness," Proc. IGARSS 1994, Pasadena, USA, pp. 1090-1092.

[8] Rott H. et al., "Cold Regions Hydrology High-Resolution Observatory for Snow and Cold Land Processes," Proc. of the IEEE, 98(5), pp. 752-765, May 2010.

[9] Schubert A. et al., "Influence of Atmospheric Path Delay on the Absolute Geolocation Accuracy of TerraSAR-X HighResolution Products," IEEE Trans. Geosci. Remote Sens, 48(2), pp. 751-758, Feb. 2010.

[10] Schubert A. et al., "Mitigation of atmospheric perturbations and solid Earth movements in a TerraSAR-X time-series," Journal of Geodesy, 86(4), 2012, pp. 257-270.

[11] Small, D., M. Jehle, E. Meier and D. Nüesch, "Radiometric Terrain Correction Incorporating Local Antenna Gain," VDE, Proc. EUSAR 2004, Ulm, Germany, pp. 929-932.
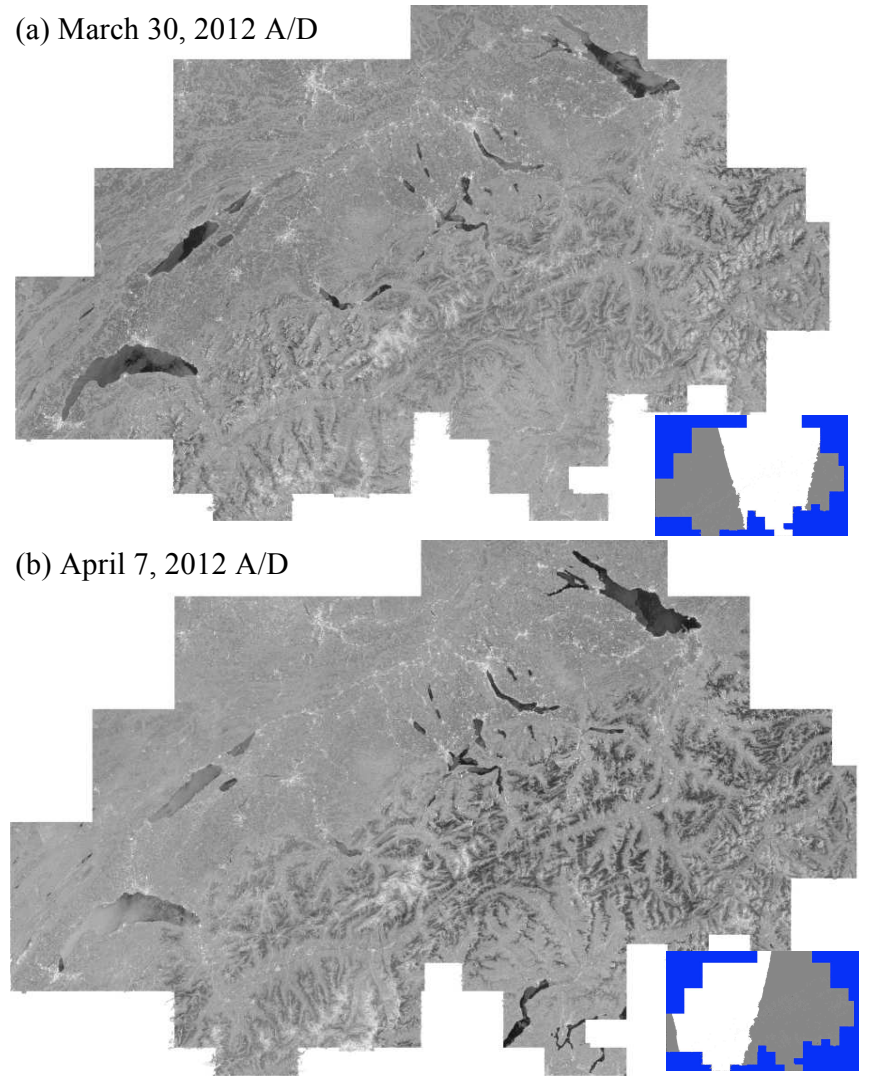

Figure 2: LRW Composites of Switzerland from ENVISAT ASAR Wide Swath VV-pol imagery (a) March 30, 2012 A/D, (b) April 7, 2012 A/D; black: -20dB, white: $+5 \mathrm{~dB}$

[12] Small, D., N. Miranda, L. Zuberbühler, A. Schubert and E. Meier, "Terrain-corrected Gamma: Improved Thematic Land-cover Retrieval for SAR with Robust Radiometric Terrain Correction," ESA SP-686, Proc. ESA Living Planet Symposium, 2010, Bergen, Norway, 8p.

[13] Small D., N. Miranda, and E. Meier, "A revised radiometric normalisation standard for SAR," Proc. IGARSS 2009, Cape Town, South Africa, pp. 566-569.

[14] Small D, "Flattening Gamma: Radiometric Terrain Correction for SAR Imagery," IEEE Trans. Geosci. Remote Sens., 49(8), pp. 3081-3093, Aug. 2011.

[15] Small D., Zuberbühler, A. Schubert, E. Meier, "Terrainflattened gamma nought Radarsat-2 backscatter," Canadian Journal of Remote Sensing, 37(5), pp. 493-499, Oct. 2011.

[16] swisstopo, "DHM25: the digital height model of Switzerland - Product Information,” June 2005, 15p.

[17] Torres R. et al., "GMES Sentinel-1 mission," Remote Sensing of Environment, Vol. 120, pp. 9-24, Feb. 2012.

[18] Ulander L., "Radiometric slope correction of synthetic aperture radar images," IEEE Trans. Geosci. Remote Sens., 34(5), pp. 1115-1122, Sep. 1996.

[19] Woodhouse I., A. Marino, and I. Cameron, "A standard index of spatial resolution for distributed targets in synthetic aperture radar imagery," Intl. Journal of Remote Sensing, 32(23), pp. 7929-7938, Dec. 2011. 